\title{
The Problem of Validity Proofs
}

\author{
Timm Lampert, Michael Baumgartner*
}

\begin{abstract}
In philosophical contexts, logical formalisms are often resorted to as a means to render the validity and invalidity of informal arguments formally transparent. Since Oliver (1967) and Massey (1975), however, it has been recognized in the literature that identifying valid arguments is easier than identifying invalid ones. Still, any viable theory of adequate logical formalization should at least reliably identify valid arguments. This paper argues that accounts of logical formalization as developed by Blau (1977) and Brun (2004) do not meet that benchmark. The paper ends by suggesting different strategies to remedy the problem.
\end{abstract}

\section{Introduction}

One of the main functions that is commonly attributed to logical formalisms in philosophical contexts consists in rendering the validity or invalidity of informal arguments transparent. The (in)validity of an argument is not unambiguously determinable based on its natural language form. Logical formalisms are widely seen to provide the theoretical fundament for systematic and reliable validity assessments. However, before formalisms can be put to work in the reconstruction of natural language arguments, the latter's component statements must be transferred into the syntax of a pertaining formalism, i.e. they must be formalized.

Theories of formalization aim to provide a systematic understanding of logical formalizations. The project of developing such theories can be (and has been) approached in three different ways: (A) by conceptually explicating the notion of formalization, (B) by specifying criteria of adequate formalization, and (C) by defining an effective formalization procedure. For reasons given in what follows we take approaches (A) and (C) to face severe problems and, hence, join authors as Blau (1977) and Brun (2004) in giving preference to approach (B). As formalizing a statement, in one way or another, amounts to representing or identifying the logical form (or the logical structure or the logical features) of the pertaining statement (cf. Borg and Lepore 2002, 99, Blau 1977, 3, Brun 2004, ch. 4), explications of the notion of formalization must, in one way or another, draw on the

\footnotetext{
${ }^{*}$ We profited a lot from intensive discussions with Georg Brun about his book and earlier drafts of this paper. Moreover, we are grateful to Sebastian Leugger, Johannes Marti, Tim Raez, and to the anonymous referees for this journal for helpful comments on an earlier draft. Finally, Michael Baumgartner would like to thank the Swiss National Science Foundation for generous support of this work (grant PP001-114812/1).
} 
the notion of logical form and, hence, presuppose clarity on that notion. Most (non-standardized) natural language statements, however, do not wear their logical forms on their sleeves. As prominently stated in the notorious misleading form thesis (cf. e.g. Brun 2004, 161-165), the syntax and grammar of ordinary statements are often radically misleading with respect to underlying logical forms. It is hence impossible to identify logical forms by drawing on syntactic properties of natural language statements. Ascribing logical forms to statements calls for stringent justification, which, in turn, must be rooted in pertaining criteria, viz. in criteria of adequate formalization. Nonetheless, approach (A), as discussed in Brun (2004, ch. 4) (cf. also Kleinknecht 2008), aims to explicate the notion of formalization independently of formalization criteria. In light of the misleading surface of natural language statements, we take this to be impossible. Instead, we are going to settle for a modest concept of formalization in this paper, according to which a logical formalization simply consists in an assignment of a logical formula to a natural language statement.

By contrast, representatives of approach (C), as Davidson (1984), Chomsky (1977), Massey (1975), or Montague (1974a), implicitly or explicitly subscribe to the ambitious project to define effective formalization procedures. Such procedures are intended to take natural language statements as inputs and to produce logical formulas as outputs. Accordingly, the misleading surface of natural language statements also constitutes a major obstacle to a successful definition of a formalization procedure that would be applicable to more than an artificially truncated fragment of a natural language or a previously disambiguated and standardized (non-natural) idiom (cf. Montague 1974c, Montague 1974b). We take this obstacle to be insurmountable. Rather than on its grammatical surface, the formalization of a natural language statement depends on the latter's informal interpretation, which is notoriously ambiguous and context dependent. In any case, however, as the quality of any formalization procedure, in the end, must be evaluated based on whether it generates adequate formalizations, the question as to the adequacy of formalizations turns out to be conceptually prior. All in all, the key project of a theory of formalization, in our view, consists in defining adequacy criteria for formalizations. Accordingly, the argument presented in this paper focuses on the criteria-driven thread in the formalization literature.

The existing literature adopting approach (B) comprises only a handful of studies, including e.g. Blau (1977), Epstein (1990, 1994), Sainsbury (1993), or recently and most thoroughly Brun (2004). ${ }^{1}$ As Brun's theory is by far the most detailed elaboration of approach (B), we base our upcoming discussion of the problems of identifying valid and invalid arguments on Brun's framework. The latter is characterized by three determinants: adequate formalizations (I) must be correct, (II) they have to be maximally faithful to the syntactic surface of the formalized statement, and (III) they must be related, i.e. any two different adequate formalizations of the same statement must either be equivalent or one must be more specific than the other or there must exist an adequate formalization that is more specific than both

\footnotetext{
${ }^{1}$ Cf. also the discussions of Brun's criteria in Löffler (2006).
} 
of them.

Theories of adequate formalization need to guarantee that logical formalisms can fulfill the functions ascribed to them. Applied to the present context, that means that adequacy criteria for logical formalizations have to enable formalisms to play their designated role in argument reconstruction. Oliver (1967) and Massey (1975), however, revealed that the common practice of formalizing arguments faces a problem when it comes to assessing the invalidity of arguments. In a nutshell, this problem of invalidity proofs stems from the fact that an argument is commonly said to be valid if and only if it is an instance of at least one valid form (Oliver 1967, 464). Consequently, an argument is invalid if and only if there exists no valid form of which that argument is an instance. That is, establishing the formal invalidity of an argument requires establishing the truth of a negative existential, which is a task that, in many cases, is difficult to complete. In contrast, revealing the validity of an argument only calls for the identification of one valid form of which that argument is an instance. Thus, demonstrating the validity of an argument is significantly easier than demonstrating an argument's invalidity. Correspondingly, while a theory of logical formalization may be acceptable even if it does not provide the means for conclusive invalidity proofs, any satisfactory account of adequate formalization must, at any rate, supply the resources to prove the validity of an argument by use of adequate formalizations. More concretely, in order to lay the theoretical fundament for formalisms to fulfill their function for validity assessments, a theory of adequate formalization must guarantee the existence of a formally valid adequate formalization for every formally valid argument.

We shall argue that Brun's account of formalization falls short of meeting this benchmark. His theory not only faces a problem of invalidity proofs but, moreover, does not guarantee that to every formally valid argument there exists at least one formally valid adequate formalization. That is, Brun's theory of formalization gives rise to what we will dub a problem of validity proofs. In consequence, if logical formalization is taken to be regulated by (I), (II), and (III), logical formalisms are no reliable tool for argument reconstruction.

\section{Brun's Criteria of Adequate Formalization}

Before we exhibit the problems of invalidity and validity proofs, this section is going to review the three tenets of Brun's theory. To this end, some conceptual preliminaries are called for. As indicated in the previous section, a modest notion of formalization suffices for the purposes of this paper, according to which a formalization is simply an assignment of a logical formula to a natural language statement relative to an instantiation. Logical formalisms provide well-known and straightforward recursive definitions of the notion of a logical formula. For simplicity, we are going to confine our discussion to formalizations in first-order for- 
malisms in the present context. ${ }^{2}$ Natural language statements are statements of natural language that are either true or false. In addition to the concepts of a logical formula and a statement, assigning logical formulas to statements presupposes assignments of natural language expressions to the categorematic parts of a formula, i.e. to the propositional variables, the proper names, and the predicate letters. Such assignments shall be dubbed instantiations. ${ }^{3}$ Finally, it is of crucial importance to note that Brun presupposes a semantics of truth-possibilities or possible extensions, rather than a semantics of truth values or (actual) extensions-as is common, for instance, in mathematical logic. While the latter assigns one fixed extension to every categorematic part of a formula and takes different such assignments to stand for different natural language expressions with different extensions, the former systematically varies assignments of extensions and interprets combinatorially varied extensions as possible extensions of one and the same statement, predicate, or name. As we shall adopt Brun's framework in the following, we not only need instantiations of formulas, but moreover assignments of possible extensions to the categorematic parts of a formula. We will label such assignments interpretations below.

The first adequacy criterion of Brun's theory is correctness, which he equivalently defines syntactically and semantically. Syntactically put, correctness of a formalization $\Phi$ of a statement $A$ amounts to the following (cf. e.g. Blau 1977, 4, Brun 2004, 214):

(COR) The formalization $\Phi$ of a statement $A$ is correct if and only if every inference $S$, such that the formalization $\Psi$ of $S$ contains $\Phi$ as a premise or a conclusion and $\Psi$ is formally valid, is informally valid.

An inference is valid if and only if, given the truth of its premises, its consequences cannot be false. Furthermore, while formal validity is to be understood on the basis of a respective calculus, an inference is said to be informally valid if it is judged to be valid without compulsory recourse to any criterion of its validity. Similarly, we often use expressions correctly without being able to define them or justify their application. In the following we use $f$-validity and $i$-validity as shorthands for formal and informal validity, respectively. By presupposing informal validity assessments, (COR) draws on the insight that it is impossible to account for adequate formalization merely based on syntactic properties of natural language statements. Thus, (COR) does justice to the misleading form thesis.

According to the standard view, arguments can be informally valid for two different reasons: an argument as "Men are mortal. Shamus is a man. Therefore, Shamus is mortal" is said to be valid on account of its plain form or syntax, whereas the validity of an argument as "Daryl is a mother. Therefore, Daryl is a woman" is taken to depend on the meanings of its constituents (cf. e.g. Davidson

\footnotetext{
${ }^{2}$ Yet, we only explicitly relativize definitions to first-order logic in this paper where this is relevant for the argumentation.

${ }^{3}$ Many alternative labels have been used in the literature, for instance, "interpretation", "key", "realization", "legend" or "correspondence schema" (cf. Brun 2004, 140).
} 
1967, Epstein 1990). To the former sort of a validity Brun $(2004,37)$ refers as informal formal validity and to the latter as informal material validity, which we subsequently abbreviate by formal $i$-validity and material $i$-validity, respectively. It is important to emphasize that, even though Brun contends that when it comes to formally proving the $i$-validity of an argument only formal $i$-validity may be adequately reproduced by $f$-valid formalizations, he does not define the correctness of a formalization with recourse to the notion of formal $i$-validity. He holds that it is not possible to sharply distinguish between formal and material $i$-validity independently of a logical formalization. Hence, the notion of formal $i$-validity cannot be presupposed when assessing the correctness of a formalization. This, however, entails that both formally and materially $i$-valid arguments are correctly reproducible by $f$-valid formalizations. To exclude that materially $i$-valid arguments can also be adequately formalized $f$-validly, Brun later introduces surface maxims-to which we shall turn below-into his theory.

Brun conceives of (COR) as a necessary condition of the adequacy of a formalization. However, as he repeatedly points out himself (chs. 11, 12), applying (COR) to concrete statements is not unproblematic. For instance, determining whether a formalization $\Phi$ is correct for a statement $A$, according to (COR), presupposes the correctness of the formalizations of the other statements involved in inferences $S$. Assessing the correctness of these latter formalizations, in turn, presupposes the correctness of $\Phi$ for $A$. Hence, applications of (COR) give rise to a circularity problem. By contrast, Brun's equivalent semantic variant of the correctness criterion is not affected by this problem, because assessing the correctness of a formalization $\Phi$ along semantic lines does not presuppose the correctness of other formalizations and, hence, is free from circularities. ${ }^{4}$ More concretely, Brun's semantic variant of correctness amounts to the following (cf. Brun 2004, 210):

(TC) The formalization $\Phi$ of a statement $A$ is correct if and only if relative to all suitable interpretations $\Im_{s}$ of $\Phi, \Phi$ has the same conditions of truth and falsehood as $A$ has according to the informal judgement.

Applying (TC) presupposes that it is determined what truth and falsehood conditions of formulas are as opposed to truth and falsehood conditions of statements and how these conditions can be compared. Subject to (TC), this comparability shall be guaranteed by suitable interpretations. Thus, two notions involved in (TC) call for clarifications: suitable interpretation and sameness of truth and falsehood conditions.

As indicated above, interpretations of a formula are assignments of possible extensions to the categorematic parts of the formula. By suitable interpretations Brun (2004, 211-212) means interpretations that are limited in extent due to informal inferential dependencies among the expressions that are assigned to the

\footnotetext{
${ }^{4}$ Both the syntactic and the semantic criterion of correctness, however, face a problem of termination when they are applied to concrete formalizations. We come back to this problem in section 5 below.
} 
categorematic symbols by a given instantiation (similarly Blau 1977). In short, suitable interpretations are a function of a corresponding instantiation. The sort of limitation involved in suitable interpretations is best clarified by concrete examples. First, consider statement (a) along with its forthright formalization (1) and instantiation (2).

(a) Daryl is a mother or Daryl is a woman.

$$
p \vee q
$$

$p$ : Daryl is a mother. ; $q$ : Daryl is a woman.

The set of suitable interpretations of (1) does not contain 4 elements as would be generated by permutationally assigning truth-possibilities, but only 3 : $\Im(p)=$ $T, \Im(q)=T ; \Im(p)=F, \Im(q)=T ; \Im(p)=F, \Im(q)=F$. On informal grounds, it is excluded that something is a mother but no woman. This informal judgement, accordingly, eliminates $\Im(p)=T, \Im(q)=F$ from the set of suitable interpretations of (1). It is this elimination that, according to Brun, allows for comparing the truth conditions of (1) and (a) in the first place and that guarantees the equivalence of the syntactic and the semantic variant of correctness. Were $\Im(p)=T, \Im(q)=F$ not discarded, we would be confronted with a configuration that informally cannot be judged coherently, because from Daryl's motherhood the truth of (a) follows, whereas Daryl not being a woman informally implies the falsehood of (a). Consequently, (TC) would be inapplicable.

The truth conditions of a formalization $\Phi$ of a statement $A$ consist in the models of $\Phi$ among its suitable interpretations, i.e. in its suitable models. By contrast, the falsehood conditions of $\Phi$ consist in its suitable counter-models. The truth and falsehood conditions of $A$, on the other hand, are generated by paraphrasing the suitable interpretations relative to a given instantiation of $\Phi$ and informally assessing the truth values of $A$ with recourse to these paraphrases. For instance, the paraphrases of the suitable interpretations of (1) in virtue of its instantiation (2) are the following: (i) "Daryl is a mother", "Daryl is a woman"; (ii) "Daryl is no mother", "Daryl is a woman"; (iii) "Daryl is no mother", "Daryl is no woman". Under conditions (i) and (ii) the statement (a) is informally judged to be true. Thus, the truth conditions of (a) are (i) and (ii). Whereas (iii) is the falsehood condition of (a), because when Daryl is neither a mother nor a woman, (a) is informally judged to be false. A comparison of the truth and falsehood conditions of a statement $A$ and its formalization $\Phi$ amounts to confronting the suitable models and counter-models of $\Phi$ with those paraphrases of the suitable interpretations that are informally seen to render $A$ true and false, respectively. By stipulating sameness of truth and falsehood conditions of $\Phi$ and $A$, (TC) demands that the paraphrases of suitable models and counter-models are informally judged to render the formalized statement true and false, respectively.

Note that (COR) and (TC) do not necessitate an adequate formalization to mirror any aspects of the inner structure of a formalized statement. According to (COR) and (TC), formalizing any statement—even a whole argument—by $p$ along 
with an appropriate instantiation is always correct (cf. Blau 1977, 7, and Brun 2004, 210, 240-241). This is due to the fact that (COR)- and (TC)-correct formalizations do not have to be complete, where a formalization $\Phi$ of $A$ is complete if and only if whatever informally follows from $A$, formally follows from $\Phi$, and whatever informally implies $A$, formally implies $\Phi$. In section 5 we shall see that one conceivable solution of the problems of (in)validity proofs consists in being more demanding with respect to completeness.

The second tenet of Brun's theory complements correctness by introducing maxims which demand that adequate formalizations reproduce the surface of formalized statements as much as possible. As anticipated above, these additional maxims requiring syntax adherence are induced by the fact that correct formalizations do not allow for distinguishing between formally and materially $i$-valid arguments, which is a distinction Brun emphatically endorses (cf. e.g. Brun 2004, 330-332). To illustrate, consider argument (b) that is classified as materially $i$ valid. (COR) and (TC) permit to formalize (b) by either of the $f$-valid implications (3), (4), or (5). ${ }^{5}$

(b) Daryl is a mother. Therefore, Daryl is a woman.

$$
\begin{gathered}
F a \wedge G a \rightarrow G a \\
F a \wedge \forall x(F x \rightarrow G x) \rightarrow G a
\end{gathered}
$$

$a:$ Daryl $; F: \ldots$ is a mother $; G: \ldots$ is a woman

$$
H a \wedge G a \rightarrow G a
$$

$a:$ Daryl $; H: \ldots$ has a child $; G: \ldots$ is a woman

In (3), the conclusion of (b) is simply introduced as an additional premise, (4) results from making the conceptual dependency of the notions of a mother and a woman formally explicit—in the vein of what Massey $(1975,66-68)$ has called the enthymematic ploy —, and in (5) the notion of a mother is semantically analyzed in terms of a woman having a child. All of these correct formalizations reproduce (b) as a formally $i$-valid argument, which, in Brun's view, it is not.

Following common formalization practice, Brun $(2004,332)$ holds that a materially $i$-valid argument as (b) should be adequately reproduced by a material implication as (6) - which is also correct for (b) because all of its counter-models are unsuitable in Brun's sense of the term.

$$
F a \rightarrow G a
$$

$$
a: \text { Daryl } ; F: \ldots \text { is a mother } ; G: \ldots \text { is a woman }
$$

To deny (3), (4), and (5) the status of adequate formalizations of (b), in particular, and to theoretically back up the distinction between formal and material $i$-validity,

\footnotetext{
${ }^{5}$ Arguments are often formalized by means of argument schemes involving operators as "†" or " $\because$ " that belong to metalanguage. As we are exclusively concerned with first-order formalizations in the present context, we dispense with metalingual operators in formalization candidates throughout this paper (cf. also Epstein 1990, 24 or Brun 2004, 46, 243).
} 
in general, a criterion is needed that identifies those $i$-valid arguments that may be reproduced by $f$-valid formalizations. For this purpose, Brun introduces surface maxims which stipulate that adequate formalizations must be maximally similar to the syntactic and grammatical surface of formalized statements. For example, (b) involves the predicates "... is a mother" and "... is a woman" and its premise does not feature a conjunction. All of the correct formalizations (3), (4), and (5) significantly deviate from this syntactic surface-(3) and (4) by supplying premises not contained in (b), and (5) by semantically analyzing "... is a mother". Of all four formalization candidates, (6) is maximally faithful to the surface of (b), and is thus preferable over (3), (4), and (5).

Basically there are two directions available in regard to explicating how formalizations mirror syntactic features of a statement: (i) a feature of a formalization should have a counterpart in the statement such that the same/different features are captured by the same/different counterparts in the statement; (ii) a feature of a statement should have a counterpart in the formalization such that the same/different features are captured by the same/different counterparts in the formalization. Both of these directions can be found in formalization strategies available in the literature. $^{6}$ Relative to (i) and (ii) it is then possible to formulate concrete surface maxims, which, for brevity, we do not reproduce in detail here. Just to give one example, based on (i) Brun $(2004,254)$ formulates the following correspondence rule (KS): The instantiation of an adequate formalization $\Phi$ of a statement $A$ only features ordinary expressions that also occur in $A$.

While the application of this rule is unproblematic, surface faithfulness is not as easily satisfiable in regard to other syntactic features of statements, such as the order of expressions or the correspondence of syncategorematic parts of a statement and of its formalization. The fact that the surface of natural language statements is often misleading as to underlying logical forms renders it inevitable, in many cases, to purposefully digress from that surface in order to correctly formalize a statement. Therefore, surface similarity in either of the senses given by (i) and (ii) must not be demanded unrestrictedly. To see that (i) cannot be applied in an unconstrained manner, consider a statement as "Men are mortal", which is normally said to be adequately captured by $\forall x(F x \rightarrow G x)$. This formula features a universal quantifier and a conditional, neither of which has a counterpart in "Men are mortal". By contrast, an unconditional application of a maxim oriented in terms of (ii) would misleadingly dictate to assign different formulas to "This argument is invalid" and "This argument is not valid". Surface similarity often can only be assessed relative to a given set of equally specific correct formalizations. While, as we shall see below, correctness can be satisfied on various levels of specification and regularly induces to depart from the surface of natural language, surface maxims require that the differences between formalizations and pertaining statements

\footnotetext{
${ }^{6}$ While Brun's (2004) surface maxims are oriented in terms of (i) (cf. also Sainsbury 1993, 69), Epstein (1994) formalizes by abstracting from the content of statements in a stepwise manner and, thus, adopts strategy (ii).
} 
be minimized.

Based on surface maxims it is now possible to specify a criterion that theoretically substantiates the distinction between formal and material $i$-validity by identifying arguments that are formally $i$-valid relative to the formalism of interest in this paper, that is, relative first-order logic. The existence of a correct $f$-valid first-order formalization $\Phi$ of an argument $S$ entails that $S$ is $i$-valid. If $\Phi$ moreover satisfies surface maxims, the $f$-validity of $\Phi$ is implied to be independent of any kind of semantic analysis of categorematic parts of $S$. This, in turn, determines that the $i$-validity of $S$ is not due to the meanings of its parts but to its mere form as given by the first-order formalism. Hence, $S$ is entailed to be an instance of an $f$-valid first-order form, i.e. to be formally $i$-valid: ${ }^{7}$

(IFVP) If there exists at least one correct and surface faithful $f$-valid first-order formalization of an argument $S, S$ is formally $i$-valid relative to first-order logic.

Traditionally, a statement $A$ is first formalized by an unspecific adequate formalization which is then gradually specified until the purposes of formalizing $A$ are met. However, as will be illustrated in section 4, correctness and surface maxims allow for correctly and surface faithfully formalizing a statement by means of manifold non-equivalent formulas none of which corresponds to a mere specification of the other. Such unrelated formalizations cannot be interpreted as expressing the same logical form. In consequence, if logical forms of statements are to be identified by drawing on formalizations, correct and surface faithful formalizations of a statement $A$ are insufficient to unambiguously assign one logical form to $A$. In other words, tenets (I) and (II) do not exclude that more than one logical form is attributed to one and the same statement.

To avoid this unwelcome consequence and to ensure the unity of logical form, Brun introduces a further adequacy criterion. Roughly, this additional criterion stipulates that all adequate formalizations of a statement $A$ must either be equivalent or mutually dependent in terms of the following relation of specification. A formula $\Phi$ is more specific than a formula $\Psi$ if and only if $\Phi$ is generated from $\Psi$ by substitutions $\alpha / \beta$ satisfying one of the following two conditions: ${ }^{8}$ (i) $\alpha$ is a propositional variable, $\beta$ is a complex propositional formula or a formula of first-order $\operatorname{logic}$; (ii) $\alpha$ is an $n$-place predicate, $\beta$ is an open formula with $n$ free variables containing at least one sentential connective, a quantifier or a predicate with more than $n$ places. For instance, as to (i) $q \vee(s \wedge t)$ is more specific than $q \vee r$ and subject to (ii) $F a b$ is more specific than $F a$ and $\exists y(\forall x F x y \wedge G y)$ is more specific than $\exists y(F y \wedge G y)$. That is, if we take propositional variables to be 0 -place predicates,

\footnotetext{
${ }^{7}$ (IFVP) must be distinguished from another criterion for formal $i$-validity that is often mentioned in textbooks and that does not draw on correct formalizations, but rather on the substitutability of nonlogical expressions within ordinary language arguments. This substitutional criterion is discussed and contrasted with (IFVP) in section 5 below.

${ }^{8}$ This definition is taken from Brun $(2004,320)$. Cf. also Kapitan $(1984,18)$ and Sainsbury $(1993$, 211-213). For a detailed discussion of formalizations that differ in specificity cf. Brun (2004, ch. 13).
} 
the only elements of a formula that can be specified are its predicates. Moreover, a specification $\Phi^{\prime}$ of a formalization $\Phi$ of an argument must respect the structure of $\Phi$, i.e. $\Phi^{\prime}$ must be an instance of the same inference scheme as $\Phi$ (cf. Brun 2004, 309-310). A specification of $\Phi$ may only introduce new substructures within the logical hierarchy of $\Phi$. Thus, for example, the main operator of $\Phi$ must remain unchanged across specifications. However, due to surface maxims the possibilities to specify a formula are not unlimited. Most of all, categorematic parts of formulas must not be specified by means of semantic analysis that introduces components not contained in the formalized statement. The narrow definition of specification is designed to ensure that if $\Phi$ is an adequate formalization of a statement $A$, then, on the one hand, any less specific formalization with an appropriate instantiation is adequate for $A$ as well, and on the other hand, any formalization that is more specific than $\Phi$ and adequate for $A$ contributes to rendering the $i$-validity of an argument containing $A$ formally transparent, if $\Phi$ contributes to rendering that $i$ validity transparent.

Against this theoretical background, the additional criterion of hierarchical structure which complements correctness and surface maxims and which, contrary to the latter, constitutes a relational criterion of adequacy can be more precisely stated (cf. Brun 2004, 349, 351, who refers to Castañeda 1975, 69):

(HSC) The formalizations $\Phi$ and $\Psi$ of $A$ are not both adequate if $\Phi$ and $\Psi$ are (i) not equivalent ${ }^{9}$, (ii) neither formalization is more specific than the other, and (iii) no formalization $\chi$ exists which is more specific than $\Phi$ and $\Psi$.

For brevity, formalizations $\Phi$ and $\Psi$ such that $\Phi$ and $\Psi$ are either equivalent or one of them is a specification of the other or there exists a formalization $\chi$ which is more specific than $\Phi$ and $\Psi$ shall be referred to as related formalizations. Within Brun's theory, (HSC) is an additional necessary adequacy criterion. It stipulates that of two correct formalizations that comply with surface maxims and are not related one must be inadequate. (HSC) does not, however, determine which of the two formalizations is to be rejected as inadequate.

\section{The Problem of Invalidity Proofs}

The merits of a theory of adequate formalization can be evaluated, inter alia, by determining whether it enables formalisms to do the work they are employed to do. In the present context, this amounts to assessing whether the theory provides a satisfactory framework to conduct validity and invalidity proofs. As anticipated in the introductory section, Oliver (1967) and Massey (1975) have shown that there exists an important asymmetry in regard to the difficulties posed by such (in)validity proofs: whereas, according to (IFVP), the existence of a correct, surface faithful, and $f$-valid first-order formalization of an argument $S$ is sufficient for the formal

\footnotetext{
${ }^{9}$ For the sake of simplicity, we subsume so called "notational variants of formalizations", cf. Brun (2004, 301-302), under the equivalent formalizations.
} 
$i$-validity of $S$ relative to first-order logic, the contraposition of (IFVP) stipulates that the non-existence of a $f$-valid first-order formalization of $S$ is necessary for $S$ not to be formally $i$-valid according to first-order logic. In other words, while the formal $i$-validity of $S$ follows from the existence of at least one $f$-valid form of which $S$ is an instance, exposing that $S$ is not formally $i$-valid requires establishing that no $f$-valid form exists of which $S$ is an instance, i.e. establishing the truth of a negative existential. As a consequence, demonstrating that an argument is not formally $i$-valid is more difficult than exhibiting its formal $i$-validity.

To illustrate this problem of invalidity proofs consider argument (c):

(c) Daryl strolls through Bologna. Therefore, there is a stroll.

In light of Davidson's (1967) analysis of action sentences, (c) is adequately formalized as follows:

$$
\begin{gathered}
\exists x(F x \wedge G x a \wedge H x b) \rightarrow \exists x F x \\
a: \text { Daryl ; b : Bologna ; F }: \ldots \text { is a stroll } ; \\
G: \ldots \text { is conducted by...; } H: \ldots \text { takes place in ... }
\end{gathered}
$$

Prior to Davidson's widely accepted proposal of how to formalize action sentences, there had not been a formalization of (c) available that had been both $f$-valid and commonly considered adequate. That is, given a formalization $\Phi$ of an argument $S$ such that $\Phi$ does not feature tautologous premises and contradictory conclusions and $\Phi$ is not $f$-valid, it may not be possible to infer that $S$ is not formally $i$-valid, because there could be a previously unthought-of more specific adequate formalization $\Psi$ which is $f$-valid after all. Indeed, except for trivial cases of formal $i$-invalidity, it may well be possible that specifying adequate formalizations leads to $f$-valid formalizations that are likewise adequate representations of the scrutinized argument. ${ }^{10}$ Nonetheless, the gravity of this problem should not be overrated. On Brun's account, the possibilities to specify formalizations are limited by surface maxims, as not any arbitrary specification generates adequate formalizations. Hence, Brun's theory provides an upper limit with respect to the specifiability of formalizations, i.e. the search space for invalidity proofs is not infinite. Still, as long as it cannot be excluded that there might exist a $f$-valid specification of an $f$-invalid formalization, the invalidity of the corresponding argument is not conclusively formally proven.

\section{The Problem of Validity Proofs}

Thus, while a theory of adequate formalization may not provide the means to conclusively prove the invalidity of arguments, a cogent account of logical formalization must, at least, lay the theoretical fundament to reliably conduct validity proofs.

\footnotetext{
${ }^{10}$ This essentially amounts to Oliver's (1967) variant of the problem of invalidity proofs. Massey (1975) bases his version of the problem on the allegedly open number of potential target formalisms. Since we limit our discussion to first-order logic from the outset, we do not further discuss the details of Massey (1975). For a criticism of Massey (1975) cf. also Bencivenga (1979).
} 
To this end, it must be excluded that there exist $f$-valid arguments that cannot, in principle, be $f$-validly reproduced by formalizations that meet the adequacy standards of the theory. That is, a theory of adequate formalization must entail (VP):

(VP) If an argument $S$ is formally $i$-valid relative to first-order logic, then there exists at least one adequate $f$-valid first-order formalization of $S$.

If an account of adequate formalization implies (VP), it is ensured that a pertaining logical formalism can fulfill its designated role for validity proofs. Validation of (VP) can, hence, be seen as a sort of quality benchmark for theories of formalization. As anticipated above, we are now going to argue that correctness, surface maxims, and the principle of hierarchical structure do not warrant the existence of an adequate $f$-valid first-order formalization for every argument that is formally $i$-valid relative to first-order logic, i.e. Brun's theory does not imply (VP). There exist formally $i$-valid arguments for which Brun's account does not supply a $f$-valid adequate formalization.

To introduce this problem of validity proofs, consider the following statement:

(d) All heads of horses are heads of animals.

This is the consequence in De Morgan's well-known argument "Because horses are animals, all heads of horses are heads of animals" which is frequently used in textbooks to motivate non-monadic first-order logic. The following are the ordinary textbook formalizations of (d). They are all correct and satisfy surface maxims:

$p$

$p:$ All heads of horses are heads of animals

$$
\forall x(F x \rightarrow G x)
$$

$F: \ldots$ is a head of a horse; $G: \ldots$ is a head of an animal

$$
\forall x(\exists y(H y \wedge I x y) \rightarrow \exists z(J z \wedge I x z))
$$

$H: \ldots$ is a horse; $I: \ldots$ is a head of $\ldots ; \quad J: \ldots$ is an animal

(10) is implied by $\forall x(H x \rightarrow J x)$, which, relative to the instantiation of (10), is a correct and surface faithful formalization of the premise of De Morgan's argument. Hence, the latter's formal $i$-validity can be proven with recourse to (10) (cf. Brun 2004, 355). Yet, there exist other formalizations of (d) which, according to Brun's standards, are equally correct and surface faithful and also yield a proof of De Morgan's argument, but which are unrelated to (10). In particular, (d) is correctly and surface faithfully formalized either in terms of (11), which has been suggested by Wengert (1974), or in terms of the formally equivalent expression (12), which so far has not been discussed in the literature:

$$
\begin{gathered}
\forall x \forall y(H y \wedge I x y \rightarrow J y \wedge I x y) \\
\forall x(H x \wedge \exists y I y x \rightarrow J x \wedge \exists y I y x)
\end{gathered}
$$

$H: \ldots$ is a horse; $I: \ldots$ is a head of ... $J: \ldots$ is an animal 
Both of these equivalent formalizations follow from $\forall x(H x \rightarrow J x)$ and, hence, prove the formal $i$-validity of De Morgan's argument-just as does (10). Still, (10) is not equivalent to (11) and (12). (10) follows from (11) and (12), but not vice versa. The truth conditions of (10) and of (11)/(12) differ insofar as (10) can be true if there is an object $c_{h}$ that satisfies $\Im(H)$ without satisfying $\Im(J)$ and an object $c_{i}$ related in terms of $\Im(I)$ to $c_{h}$ and some other object $c_{j}$ that satisfies $\Im(J)$, whereas all interpretations of this type render (11) and (12) false. For instance, $\Im(H)=\left\{c_{1}\right\} ; \Im(J)=\left\{c_{2}\right\} ; \Im(I)=\left\{\left(c_{3}, c_{1}\right),\left(c_{3}, c_{2}\right)\right\}$ is a model of $(10)$ and a counter-model of $(11) /(12)$. More colloquially put, (10) is true if there is a horse which is not an animal but shares its head with an animal, whereas this configuration renders (11) and (12) false. Generally, all interpretations of (10), (11), and (12) that yield differences in truth conditions involve at least one horse that is not an animal. In light of the fact that the natural language notion of a horse is defined in terms of the notion of an animal, therefore, all interpretations that distinguish among these formalizations are unsuitable in Brun's sense of the term. That means when it comes to assessing the correctness of (10), (11), and (12) for (d), all of these difference-making interpretations must be discarded. In consequence, (10), (11), and (12) are all correct for (d) according to Brun's theory. Furthermore, there is no significant difference between (10), (11), and (12) in regard to surface similarity with (d). All of these formalizations rely on the same instantiation that only features expressions that also occur in (d). They all introduce quantifiers and a subjunctor which do not have counterparts in the corresponding natural language statements. Thus, the conclusion in De Morgan's argument is surface faithfully reproduced by one of (10), (11), and (12) if and only if it is surface faithfully reproduced by all of (10), (11), and (12). ${ }^{11}$ (10) is commonly taken to be surface similar enough to (d), and Brun $(2004$, chs. 11,12$)$ explicitly acknowledges that both (10) and (11) satisfy surface maxims as well. For these reasons we conclude that, on Brun's account, (d) can be equally correctly and surface faithfully formalized by (10), (11), and (12).

While (11) and (12) are equivalent, (10) is not related to (11)/(12), for it is not equivalent to the latter, neither formalization is a specification of the other and there is no formalization $\chi$ that is more specific than (10) as well as $(11) /(12)$ (for details cf. Brun 2004, 353). In addition, (10) and (12) are both specifications of (9). Hence, unspecific adequate formalizations such as $p$ can be specified along various unrelated paths which all feature correct and surface faithful formalizations and which, thus, ascribe different logical forms to formalized statements. The tree of figure 1 graphically reproduces the two specification paths for our example. All

\footnotetext{
${ }^{11}$ Note that (11) and (12) are also equivalent to (13) and (14), which are to be understood relative to the instantiation given for (11)/(12) above:

$$
\begin{aligned}
& \neg \exists x \exists y(H y \wedge I x y \wedge \neg J y) \\
& \forall y(H y \wedge \exists x I x y \rightarrow J y)
\end{aligned}
$$

Yet, as neither of these expressions satisfy surface maxims with respect to (d) they are no viable formalization candidates based on Brun's theory. 


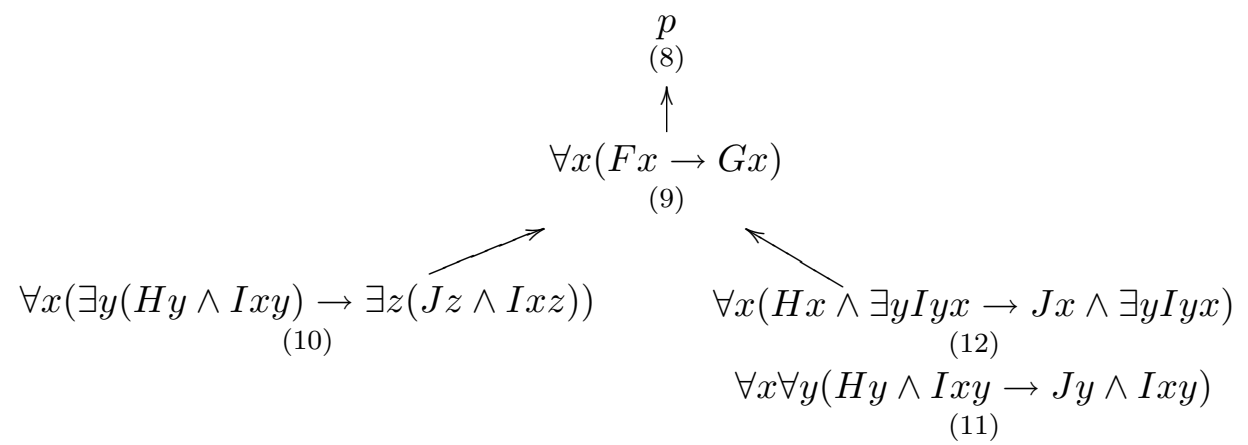

Fig. 1: Specification paths relating (8) to (12).

of this shows that correctness and surface maxims are not sufficient to guarantee the unity of logical form. As indicated in section 2, Brun fills this gap by means of (HSC). Subject to (HSC), (10) and (11)/(12) cannot both be adequate formalizations of (d). Yet, (HSC) does not determine which of (10) and (11)/(12) is to be considered as inadequate.

Prima facie, it might appear that the question as to which of (10) and (11)/(12) to choose as adequate representation of (d), in fact, is of minor importance only. For, as indicated above, both formalizations allow to capture the formal $i$-validity of De Morgan's argument "Because horses are animals, all heads of horses are heads of animals". In the literature preference is generally given to (10). While only very few authors - as Brun and Wengert — consider the possibility of formalizing (d) in terms of (11), the equally correct and surface faithful formalization candidate (12) has never been discussed before. Owing to his neglect of (12), Brun $(2004,355)$ justifies the general preference of (10) over (11) in the literature along the following lines: as (d) has the form "All $F$ s are $G$ s" which is traditionally formalized by universally quantified conditionals, (9) is unquestionably adequate for (d); therefore, since (10) is a specification of (9) whereas (11) is not, only (10) ascribes the form "All Fs are Gs" to (d) and is, hence, preferable over (11). In light of (12), though, Brun can no longer account for the general preference of (10) in this way, for (12) is also a specification of (9) and, thus, also ascribes the form "All Fs are Gs" to (d). In sum, (10) and (12)/(11) perform equally well on all counts. No tenet of Brun's theory provides any reason to give preference to (10) or $(11) /(12)$.

The choice between (10) and (11)/(12) can only be left undetermined if an arbitrary choice does not endanger the existence of a $f$-valid adequate formalization for formally $i$-valid arguments involving (d). However, there in fact are formally $i$-valid arguments containing (d) that can only be $f$-validly formalized by drawing on one of (10) and (11)/(12). In what follows we substantiate this claim by both presenting an argument whose formal $i$-validity can only be reproduced by drawing on (11)/(12) and an argument that requires recourse to (10).

First, consider (e):

(e) All heads of horses are heads of animals. The horse Fury has a head. Therefore, Fury is an animal. 
According to Brun's standards, (e) is $i$-valid, because to render its premises true and its conclusion false it is required to assume that there are horses that are no animals, which results in unsuitable conditions of truth and falsehood (Brun 2004, 231). Moreover, provided that the second premise is correctly and surface faithfully formalized by $H a \wedge \exists x I x a$ and the conclusion by $J a$-as Brun $(2004,230)$ presumes-(e)'s $i$-validity is identified to be of formal nature by (IFVP). For reproducing (e)'s first premise by the correct and surface faithful formalizations (11) or (12) yields the following $f$-valid formalizations of (e) which satisfy correctness and surface maxims:

$$
\begin{aligned}
(\forall x \forall y(H y \wedge I x y \rightarrow J y \wedge I x y) & \wedge H a \wedge \exists x I x a) \rightarrow J a \\
(\forall x(H x \wedge \exists y I y x \rightarrow J x \wedge \exists y I y x) & \wedge H a \wedge \exists x I x a) \rightarrow J a
\end{aligned}
$$

$H: \ldots$ is a horse; $I: \ldots$ is a head of $\ldots ; \quad J: \ldots$ is an animal $a:$ Fury

The fact that the $i$-validity of (e) is provable by correct and surface faithful formalizations as $\left(11^{*}\right)$ and $(12 *)$ that do not semantically analyze the categorematic expressions "head", "horse", and "animal" entails that the $i$-validity of (e) is not of material nature. $\left(11^{*}\right)$ and $\left(12^{*}\right)$ satisfy the antecedent of (IFVP) which, in turn, establishes the formal $i$-validity of (e). However, (e)'s formal $i$-validity can only be proven by formalizing its first premise, i.e. (d), by (11) or (12). More specifically, while $\left(11^{*}\right)$ and $\left(12^{*}\right)$ are $f$-valid, $\left(10^{*}\right)$ is not:

$$
(\forall x(\exists y(H y \wedge I x y) \rightarrow \exists z(J z \wedge I x z)) \wedge H a \wedge \exists x I x a) \rightarrow J a
$$

$H: \ldots$ is a horse; $I: \ldots$ is a head of $\ldots ; \quad J: \ldots$ is an animal $a:$ Fury

Of the three formalization candidates $\left(10^{*}\right)$ and $\left(11^{*}\right) /\left(12^{*}\right)$, only the latter prove the formal $i$-validity of (e). In addition, it is impossible to further specify $\left(10^{*}\right)$ in a way that results in a $f$-valid and adequate formalization of (e), because the instantiation of $\left(10^{*}\right)$ already exposes all predicates and names that are contained in (e). In consequence, $\left(10^{*}\right)$ can only be further specified by semantically analyzing the notion of a horse or of an animal or by introducing elements that do not have counterparts in (e), for example, by using the enthymematic ploy. As shown in section 2 , however, such specifications would violate surface maxims and would, hence, not be adequate.

Second, take argument (f):

(f) Everything is a head of an animal. Therefore, all heads of horses are heads of animals.

Based on our familiar instantiation used above, (f)'s premise is correctly and surface faithfully formalized by $\forall x \exists y(J y \wedge I x y)$. This formula implies (10), but it does not imply $(11) /(12)$. More specifically, while $\left(10^{* *}\right)$ is $f$-valid, $\left(11^{* *}\right)$ and $\left(12^{* *}\right)$ are not, and no specification of $\left(11^{* *}\right) /\left(12^{* *}\right)$ will generate an adequate $f$-valid formalization.

$$
\forall x \exists y(J y \wedge I x y) \rightarrow \forall x(\exists y(H y \wedge I x y) \rightarrow \exists z(J z \wedge I x z))
$$




$$
\begin{gathered}
\forall x \exists y(J y \wedge I x y) \rightarrow \forall x \forall y(H y \wedge I x y \rightarrow J y \wedge I x y) \\
\forall x \exists y(J y \wedge I x y) \rightarrow \forall x(H x \wedge \exists y I y x \rightarrow J x \wedge \exists y I y x) \\
H: \ldots \text { is a horse; } I: \ldots \text { is a head of } \ldots ; \quad J: \ldots \text { is an animal }
\end{gathered}
$$

Thus, while in case of (e) the formalizations comprising (11)/(12) are $f$-valid, it is the formalization featuring (10), i.e. $\left(10^{* *}\right)$, which is $f$-valid in case of (f). Furthermore, the validity of (f) cannot be proven by means of $\left(11^{* *}\right) /\left(12^{* *}\right)$ or specifications thereof that are adequate formalizations. Nonetheless, presuming that $\left(10^{* *}\right)$ is a correct and surface faithful formalization entails, by virtue of (IFVP), that (f) is formally $i$-valid-just as (e).

To sum up, arguments (e) and (f) both feature the statement (d), i.e. "All heads of horses are heads of animals", and both of these arguments must be taken to be formally $i$-valid on the basis of (IFVP). In order to formally reproduce the validity of (e) and (f), (d) must, in case of (e), be formalized in terms of (11)/(12) and, in case of (f), in terms of (10). Yet, (10) and (11)/(12) are not related formalizations. According to (HSC), therefore, only one of them can be adequate for (d). Our discussion above demonstrates that, which ever is chosen, there will be at least one formally $i$-valid argument that cannot be adequately formalized by a $f$-valid formalization according to Brun's theory. The latter hence does not imply (VP). That is, correctness, surface maxims, and the principle of hierarchical structure do not validate (VP). Brun's theory faces a problem of validity proofs.

To see this problem in more detail, let us consider its premises separately:

(P1) Subject to (IFVP) and to the correctness and surface faithfulness of $\left(11^{*}\right) /\left(12^{*}\right)$ and $\left(10^{* *}\right)$, (e) and (f) are formally $i$-valid arguments featuring (d) as premise or conclusion.

(P2) To formally reproduce the formal $i$-validity of (e), (d) must be reproduced in terms of the correct and surface faithful formalizations (11) or (12).

(P3) To formally reproduce the formal $i$-validity of (f), (d) must be reproduced in terms of the correct and surface faithful formalization (10).

(P4) (10) and (11)/(12) are unrelated formalizations in Brun's sense of the term.

(P1) to (P4) have the following consequences:

(C1) From (P4): According to (HSC), (10) and (11)/(12) cannot both be adequate for (d).

(C2) From (C1): It is excluded that $\left(10^{*}\right)$ and $\left(10^{* *}\right)$, which comprise (10), as well as $\left(11^{*}\right) /\left(12^{*}\right)$ and $\left(11^{* *}\right) /\left(12^{* *}\right)$, which comprise $(11) /(12)$, are adequate for (e) and (f), respectively.

(C3) From (P1), (P2), (P3), (C2): For one of the formally $i$-valid arguments (e) and (f) there does not exist an adequate $f$-valid formalization according to Brun's theory.

(C4) From (C3): Brun's theory does not imply that for every formally $i$-valid argument there exists an adequate $f$-valid formalization. 
The problem of validity proofs shows that a theory of adequate formalization that turns on correctness, surface maxims, (IFVP), and (HSC) does not warrant the existence of a $f$-valid adequate formalization for every formally $i$-valid argument. Correctness and surface maxims allow to specify formalizations of formally $i$-valid arguments as (e) and (f) along unrelated paths, one of which does not ultimately provide a proof of the argument. Yet, only one of these paths can be constituted by adequate formalizations according to (HSC). Hence, Brun's account does not meet the benchmark of providing criteria of adequate formalization which exclude that there exist formally $i$-valid arguments that cannot, in principle, be $f$-validly reproduced by formalizations that meet the adequacy standards of the pertaining theory.

\section{Conceivable Ways Out}

As the argument presented in the previous section is based on a number of premises, there are several ways to block it—some less, some more promising. In what follows we first discuss three ways around the problem of validity proofs that we do not take to be viable, and then two ways which, on our view, both feasibly solve the problem.

First, the problem of validity proofs could be avoided by rejecting tenet (III) of Brun's theory, i.e. by denying (HSC) the status of a necessary condition of adequate formalization. In consequence, (C1), which draws on (HSC) to establish that (10) and (11)/(12) cannot both be adequate for (d), is not derivable any longer. Upon rejecting (HSC), correctness and surface maxims are rendered sufficient for adequacy. Such an account is immune to the problem of validity proofs, for, under its terms, every correct and surface faithful $f$-valid formalization of an argument $S$ not only establishes the argument's formal $i$-validity but is automatically adequate for $S$. Hence, such a weakening of Brun's notion of an adequate formalization clearly validates (VP). At the same time, however, it allows for ascribing several unrelated logical formulas to one and the same statement or argument and, thus, violates the unity of logical form. For example, according to this weakening of Brun's account the formal $i$-validity of (e) and (f) are grounded in different unrelated logical formulas of (d), which in itself does not involve ambiguities. An account of formalization that does not guarantee the unity of logical form introduces ambiguities that are not contained in formalized statements, and, hence, subverts the very reason why formalisms are resorted to in the first place. Therefore, simply rejecting tenet (III) of Brun's account is no viable option.

The second unfeasible way of avoiding the problem of validity proofs amounts to rejecting (IFVP) in favor of a validity principle that is often mentioned in textbooks (cf. e.g. Bolzano 1837, §148, Salmon 1973, 18-22, Hoyningen-Huene 1998, 18-21) and that, roughly, renders formal $i$-validity dependent on persistence of $i$-validity across substitutions of non-logical expressions. This substitutional principle presupposes that natural language expressions can be divided into logical and 
non-logical ones. Relative to first-order logic, the former category comprises expressions such as "not", "and", "all", "some" etc. and the latter contains all other expressions. Moreover, non-logical terms must be grouped into proper substitution classes all of whose elements can be mutually substituted without violating the grammar of the pertaining language. By referring to such substitutions as $F O L$ substitutions, the substitutional validity principle can be more precisely stated:

(IFVP $_{s u b}$ ) If an $i$-valid argument $S$ remains $i$-valid across all FOL-substitutions, $S$ is formally $i$-valid relative to first-order logic.

Contrary to (IFVP), (IFVP sub $)$ aims to identify formal $i$-validity based on the natural language surface of pertaining arguments. Plainly, the division of natural language expressions into logical and non-logical ones and the grouping of the latter into proper substitution classes based on mere grammatical criteria raise highly intricate questions. In light of the misleading surface of natural language, it is far from clear that these questions can be answered in a satisfactorily general manner. Nonetheless, $\left(\mathrm{IFVP}_{s u b}\right)$ could be argued to pave the way around the problem of validity proofs, because, unlike (IFVP), $\left(\mathrm{IFVP}_{\text {sub }}\right.$ ) does not allow for establishing the formal $i$-validity of (e). Substituting non-logical expressions in (e) does not preserve $i$-validity, as the following $i$-invalid substitution instance shows:

(g) All children of mothers are children of fathers. The mother Ann has a child. Therefore, Ann is a father.

Even though $\left(\mathrm{IFVP}_{\text {sub }}\right)$ can hence be taken to eliminate the justificatory basis of (P1), it does not persuasively solve the problem of validity proofs presented in the previous section, because $\left(\mathrm{IFVP}_{s u b}\right)$ immediately gives rise to another (epistemic) problem of validity proofs, which is directly analogous to the problem of invalidity proofs. As Salmon $(1973,20-21)$ points out, in order to establish the formal $i$-validity of an argument $S$ based on $\left(\mathrm{IFVP}_{\text {sub }}\right)$, it would have to be ascertained that all FOL-substitutions preserve $i$-validity. Such a universal claim can never be conclusively verified. Moreover, while in case of the problem of invalidity proofs the specifiability of formalizations is limited by surface maxims, the possibilities for FOL-substitutions are completely unlimited. Natural language regularly allows for unexpected substitutions that must be seen to cast doubts on the formal $i$-validity of arguments according to (IFVP $\left.\mathrm{P}_{s u b}\right)$. To illustrate, consider the following argument-which is just one among many well-known analogous examples that can be found in the literature (cf. Sainsbury 2001, 44-53):

(h) Human beings are sensitive to pain. Harry is a human being. Therefore, Harry is sensitive to pain.

This is an instance of the seemingly $i$-valid schema " $F$ s are $G$ s. $\alpha$ is an $F$. Therefore, $\alpha$ is a $G$ " (cf. Sainsbury 2001, 50). However, the following invalid argument is also an instance of this schema:

(i) Human beings are evenly distributed over the earth's surface. Harry is a human being. Therefore, Harry is evenly distributed over the earth's surface. 
This example shows that the unconfined substitutability of non-logical expressions does not create a mere theoretical problem for the identifiability of $i$-validity based on $\left(\operatorname{IFVP}_{s u b}\right)$. Rather, with recourse to $\left(\operatorname{IFVP}_{s u b}\right)$, no argument can in fact be identified as formally $i$-valid.

A natural way to block the substitution that leads from (h) to (i) would, of course, be to argue that the first premise of (h) has the form of a universally quantified conditional, whereas the first premise of (i) does not. This amounts to claiming that "... are sensitive to pain" is a first-order predicate and "... are evenly distributed over the earth's surface" is of second order and that, accordingly, the two predicates are not contained in the same substitution class. Clearly, though, this difference between (h) and (i) cannot be read off the latter's surface, but calls for recourse to logical formalizations. The friend of $\left(\mathrm{IFVP}_{\text {sub }}\right)$ who wants to identify formal $i$-validity on the mere basis of natural language surface, hence, cannot block the transition from (h) to (i) in this vein.

Examples like (h) reveal that persistence of $i$-validity over all FOLsubstitutions not only falls short of singling out formally $i$-valid arguments in a finite number of steps, such persistence cannot be considered necessary for formal $i$-validity either, for otherwise (h) would mistakenly be determined to be formally $i$-invalid. Correspondingly, (e) is not demonstrated to be $i$-invalid in light of (g). Based on the surface of ordinary statements it is neither possible to identify valid nor invalid arguments. As Sainsbury (1993, 36-53) indicates, identifying logical forms by schematizing ordinary statements is a futile endeavor. He convincingly argues that the project of logical formalization starts with a logical formalism and analyzes the form of statements in the light of such a formalism. Only a criterion of formal $i$-validity that draws on the validity of logical formalizations, such as (IFVP), does justice to this finding.

The third unfeasible approach to block the argument of the previous section follows Brun in identifying formally $i$-valid arguments by means of logical formalizations and accepts Brun's adequacy criteria. Instead, it rejects (IFVP) in favor of a principle such as $\left(\mathrm{IFVP}_{a d q}\right)$ :

$\left(\operatorname{IFVP}_{a d q}\right)$ If there exists at least one adequate $f$-valid first-order formalization of an argument $S, S$ is formally $i$-valid relative to first-order logic.

In contrast to (IFVP), ( IFVP $_{a d q}$ ) stipulates that formal $i$-validity not only depends on compliance with correctness and surface maxims but also with (HSC). That, in turn, means that in order for an $f$-valid formalization $\Phi$ of $S$ to warrant the formal $i$-validity of $S, \Phi$ must be correct for $S$, faithful to the surface of $S$, and there must not exist an unrelated formalization $\Psi$ that equally satisfies correctness and surface maxims with respect to $S$. As a direct consequence, (P1) turns out false: not both (e) and (f) can possibly be formally $i$-valid subject to (IFVP $\left.{ }_{a d q}\right)$. Thereby, the problem of validity proofs is averted. Yet, this way around the problem is not viable either. On the one hand, Brun's account provides no indication whatsoever which of (e) and (f) in fact is the formally $i$-valid argument. The formal $i$-(in)validity of (e) and (f) would hence be undeterminable in principle. On the 
other hand, (IFVP ${ }_{a d q}$ ) renders the formal $i$-validity of an argument $S$ dependent on the truth of a negative existential, more specifically, on the non-existence of unrelated correct and surface faithful formalizations of $S$, which cannot be established conclusively. Thus, just like $\left(\mathrm{IFVP}_{s u b}\right),\left(\mathrm{IFVP}_{a d q}\right)$ immediately gives rise to an (epistemic) problem of validity proofs.

The above criticism of $\left(\mathrm{IFVP}_{s u b}\right)$ and $\left(\mathrm{IFVP}_{a d q}\right)$ might be countered by insisting that (IFVP) induces an epistemic problem of validity proofs as well. According to (IFVP), the identification of formally $i$-valid arguments is dependent on the availability of correct formalizations, which also requires establishing the truth of universal claims: a formalization $\Phi$ is (TC)-correct for a statement $A$ if and only if all (infinitely many) suitable models of $\Phi$ are truth conditions of $A$. Yet, as Brun (2004, 211-212) argues, the truth of this universal claim is finitely determinable by making use of finite structural descriptions of infinite classes of interpretations. For instance, the models of a formula as $\forall x(F x \rightarrow G x)$ do not need to be enumerated separately but can be described structurally: the extension of $F$ is a subset of the extension of $G, \Im(F) \subseteq \Im(G)$. In fact, our identification of unsuitable interpretations that are models of (10) and counter-models of (11)/(12) (cf. sect. 4) also makes use of such descriptions. Generally, a semantics of possible extensions identifies models and counter-models by means of structural considerations over a domain of stipulatory objects such as $c_{1}, c_{2}, \ldots$, rather than by enumerating real circumstances that render statements true or false. If the conditions of truth and falsehood of a corresponding statement coincide with respect to such structural descriptions, the application of (TC) terminates by attesting correctness. ${ }^{12}$ Accordingly, contrary to $\left(\mathrm{IFVP}_{s u b}\right)$ and $\left(\mathrm{IFVP}_{a d q}\right)$, the formal $i$-validity of an argument can be assessed in a finite number of steps on the basis of (IFVP).

Let us now turn to more promising solutions of the problem of validity proofs. In our view, the problem of validity proofs ultimately stems from Brun's weak notion of correctness, more specifically, from the fact that a formalization $\Phi$, for Brun, is correct for a natural language statement $A$ if and only if $\Phi$ and $A$ have coinciding truth conditions relative to suitable interpretations of $\Phi$ only. If none of the combinatorially possible interpretations of $\Phi$ are discarded when assessing $\Phi$ 's correctness, the problem of validity proofs can be avoided without unwelcome ramifications.

The correctness criterion can be properly strengthened by adopting one of the following strategies: Either (i) natural language expressions that are assigned to categorematic parts of formulas by pertaining instantiations are treated as if they were informally independent when it comes to comparing truth conditions of formulas and formalized statements, or (ii) logically formalizing a statement $A$ is accompanied by a semantic analysis that resolves all semantic dependencies among elements of $A$ by virtue of informally independent expressions-and, thus, disregards surface maxims-, to the effect that every interpretation of correspond-

\footnotetext{
${ }^{12}$ Lampert (2006) provides a general account of how such descriptions are generated by means of equivalence transformations to distributive normal forms within first-order logic.
} 
ing formalization candidates is inherently informally suitable. While strategy (i) shares Brun's aim to keep semantic analysis out of logical formalization, strategy (ii) takes semantic analysis to be an integral part of logical formalization. In contrast to Brun's approach, both of these strategies respect the autonomy of a logical formalism, which specifies truth conditions of formulas independently of instantiations. Let us discuss each of these strategies by applying them to the two exemplary arguments presented in the previous section.

Strategy (i) requires that all informal dependencies among natural language expressions contained in the instantiations of (10) and (11)/(12) are ignored when assessing their correctness for (d). First of all, this amounts to treating the notions of a horse and of an animal as logically independent, i.e. to assuming that horses are not defined as a subspecies of animals. While such an independence assumption may be non-natural, it has the advantage of bringing out the difference between the formalization candidates (10) and (11)/(12). To this end, interpretations as the following are of particular importance (cf. section 4): All elements in the extension ascribed to "horse" that have a head share their head with an element in the extension of "animal", but not all horses with a head are also animals with a head. Call such interpretations $\Im_{d}$, for short. Relative to $\Im_{d},(11) /(12)$ are false, whereas (10) is true. (d), in turn, is a statement about the relationship between the heads of the objects in the extension of "horse" and the heads of the objects in the extension of "animal". (d) is silent about the relationship between horses with a head and animals with a head. Therefore, provided that the notions of a horse, of an animal, and of a head are treated as if they were informally independent, (d) can be said to be true as long as there is no head of a horse which is not at the same time a head of an animal, irrespectively of whether all horses with a head are animals with a head. That is, as long as $\Im_{d}$ are not disregarded as unsuitable, only (10) can count as correct for (d). In contrast, (11)/(12) are correct formalizations of a statement as (j):

(j) All horses with a head are animals with that head.

Contrary to (d), (j) asserts that all horses that share their heads with animals are animals. Therefore, relative to a constellation as described in $\Im_{d}$, (d) can still be considered true, whereas (j) cannot. In consequence, (10) turns out to be correct for (d) and not for (j), while (11)/(12) are correct for (j) and incorrect for (d). That is, in contrast to Brun's correctness criterion, strategy (i) does not treat (d) and (j) as informally equivalent and renders that non-equivalence formally transparent by formalizing the two statements in terms of different formalizations.

Furthermore, strategy (i) induces a different informal assessment of the $i$ validity of argument (e). If the notions of a horse and an animal are treated as if they were informally independent, the mere fact that the horse Fury has a head and all heads of horses are heads of animals does not imply that Fury is also an animal. For according to that independence assumption, all heads of horses being heads of animals is compatible with some horses not being animals. Hence, strategy (i) induces a rejection of the $i$-validity of (e), and, correspondingly, calls for 
formalizing (e) in terms of the material implication (10*). By contrast, based on the aforementioned difference in truth conditions between (d) and (j) it holds that everything being a head of an animal entails that all heads of horses are heads of animals. That is, according to strategy (i), argument (f) still is formally $i$-valid and, hence, is adequately formalized by $\left(10^{* *}\right)$. In sum, strategy (i) does not give rise to a problem of validity proofs, for all arguments that are formally $i$-valid according to the standards of strategy (i) can be reproduced by $f$-valid formalizations that are adequate according to the standards of strategy (i). More specifically, strategy (i) avoids the problem of validity proofs by rejecting (P1): argument (e) is not $i$-valid.

Strategy (ii), in turn, does not require rejecting the $i$-validity of one of the arguments (e) and (f) nor does it call for ignoring informal dependencies among natural language expressions contained in instantiations. Rather, it adopts what Sainsbury (1993, ch. 6.2) has dubbed the Tractarian view, according to which any kind of necessity or impossibility must be reducible to logical necessity or impossibility (cf. Wittgenstein 1995, 6.375). Correspondingly, it aims to reduce all informal dependencies to formal ones. According to this strategy, a statement as (d) misleadingly suggests - by use of different expressions - that the notion of a horse and of an animal are logically independent, which in fact they are not. A correct logical analysis of natural language exhibits such logical dependencies as formal dependencies. Resolving all informal dependencies in this vein requires that instantiations may ascribe expressions to categorematic parts of a formula that are not mentioned in the corresponding statement. That means Brun's surface maxims must be weakened. Strategy (ii) then stipulates that instantiations only feature informally independent expressions to which all logically possible extensions can be ascribed by interpretations, none of which are informally unsuitable. Thus, the correctness of formalizations is not assessed relative to suitable interpretations that are functions of instantiations, but, rather, relative to suitable instantiations that fit the standard of logical independence. Against this background, in order for a formalization $\Phi$ to be correct for a statement $A$, i.e. to have the same conditions of truth and falsehood as $A, \Phi$ must formally reproduce all informal dependencies among the components of $A$, without distinguishing between formal and material dependencies. That is, strategy (ii) dismisses the distinction between material and formal $i$-validity. Arguments are either informally valid or invalid tout court.

To make things more concrete, the notion of a horse may, for instance, be analyzed in terms of its genus proximus "... is an animal" and a differentia specifica such as "... has four single-toed hooves". Relative to such an analysis, the fact that a statement as the premise in De Morgan's argument- "All horses are animals"expresses an analytic truth, i.e. an informal tautology, can be captured by formalizing it in terms of the formal tautology $\forall x(G x \wedge J x \rightarrow J x)$, where $G$ stands for "... has four single-toed hooves" and $J$ for "... is an animal". Moreover, in light of the fact that all horses are animals for conceptual reasons, it is also informally tautologous that all heads of horses are heads of animals, i.e. (d) is an analytic truth as well. Accordingly, strategy (ii) formalizes (d) by a formal tautology that captures the analytic relation between horses and animals. On the basis of the above 
analysis, the following formalization then turns out to be correct for (d):

$$
\forall x(G x \wedge J x \wedge H x \rightarrow J x \wedge H x)
$$

$G: \ldots$ has four single-toed hooves; $J: \ldots$ is an animal; $H: \ldots$ has a head

Rendering all informal dependencies formally transparent, as suggested by strategy (ii), yields that both the premise and the conclusion in De Morgan's argument are tautologous. In virtue of such an understanding, De Morgan's argument does not require a non-monadic formalization. Its premise merely expresses the implicit presumption that horses are defined by their genus proximus, i.e. by their being animals. This presumption must be made explicit in the course of formalizing statements on horses and animals. In light of the analyticity of its premise, its conclusion also turns out to express an analytic truth which must be formalized by a formal tautology that renders the analytic relation between horses and animals transparent. This is just what (15) explicates.

As (d) is tautologous by virtue of the standards of strategy (ii), it is irrelevant to the validity of (e). The validity of (e) entirely depends on its second premise. (f), in turn, is $i$-valid because any informal tautology such as (d) is implied by any statement. Correspondingly, (15) is entailed by any formula. According to strategy (ii), De Morgan's argument, (e), and (f) are all $i$-valid arguments whose $i$ validity essentially draws on the analytic truth "All horses are animals". (15) allows for straightforwardly reproducing the $i$-validity of these arguments by virtue of $f$ valid implications. In sum, strategy (ii) avoids the problem of validity proofs by rejecting (P2) and (P3): By taking seriously the fact that "All horses are animals" is an analytic truth and that, accordingly, (d) is informally tautologous as well, both (10) and (11)/(12) must be rejected as adequate formal reproductions of (d).

Strategy (ii) not only demands that informally valid arguments correspond to valid formalizations but but also that valid formalizations correspond to informally valid arguments. Correct formalizations must mirror all informal dependencies among the elements of formalized texts. With respect to these informal dependencies, thus, strategy (ii) demands completeness as a constraint on correct formalizations. That is, the standards of strategy (ii) support the following principle of invalidity: An argument $S$ is informally invalid if and only if there exists at least one adequate invalid formalization of $S$. Contrary to strategy (i), therefore, strategy (ii) not only avoids the problem of validity proofs but also the problem of invalidity proofs.

\section{References}

Bencivenga, E. (1979). On good and bad arguments. Journal of Philosophical Logic 8, $247-259$.

Blau, U. (1977). Die dreiwertige Logik der Sprache. Berlin: de Gruyter.

Bolzano, B. (1837). Wissenschaftslehre, Volume 4 vol. Sulzbach: Seidel.

Borg, E. and E. Lepore (2002). Symbolic logic and natural language. In D. Jacquette (Ed.), A Companion to Philosophical Logic, pp. 86-101. Oxford: Blackwell. 
Brun, G. (2004). Die richtige Formel. Philosophische Probleme der logischen Formalisierung. Frankfurt a.M.: Ontos.

Castañeda, H.-N. (1975). Thinking and doing. The philosophical foundations of instituitions. Dordrecht: Reidel.

Chomsky, N. (1977). Essays on form and interpretation. Amsterdam: North-Holland.

Davidson, D. (1967). The logical form of action sentences. In N. Rescher (Ed.), The Logic of Decision and Action, pp. 81-95. Pittsburgh: University of Pittsburgh Press.

Davidson, D. (1984). Inquiries into truth and interpretation. Oxford: Oxford University Press.

Epstein, R. L. (1990). The Semantic Foundations of Logic: Propositional Logic. Dordrecht: Kluwer.

Epstein, R. L. (1994). The Semantic Foundations of Logic: Predicate Logic. Oxford: Oxford University Press.

Hoyningen-Huene, P. (1998). Formale Logik. Stuttgart: Reclam.

Kapitan, T. (1984). Form and implication. Logique et analyse 27, 15-38.

Kleinknecht, R. (2008). Probleme des Formalisierens: Zum Verhältnis zwischen natürlichen und formalen Sprachen. In G. Kreuzbauer, N. Gratzl, and E. Hiebl (Eds.), Rhetorische Wissenschaft: Rede und Argumentation in Theorie und Praxis, pp. 163-178. Wien: LIT-Verlag.

Lampert, T. (2006). Explaining formulae of first order logic. Ruch Filozoficzny LXIII.3, 459-480.

Löffler, W. (2006). Spielt die rhethorische Qualität von Argumenten eine Rolle bei deren logischer Analyse? Überlegungen zum Verhältnis von Argumentationstheorie und formaler Logik. In G. Kreuzbauer and G. Dorn (Eds.), Argumentation in Theorie und Praxis (Salzburger Beiträge zu Rhetorik und Argumentationstheorie, Band 1), pp. 115-130. Wien: LIT.

Massey, G. J. (1975). Are there any good arguments that bad arguments are bad? Philosophy in Context 4, 61-77.

Montague, R. (1974a). Formal Philosophy: Selected Papers of Richard Montague. New Haven: Yale University Press.

Montague, R. (1974b). Quantification in ordinary language. In R. H. Thomason (Ed.), Formal Philosophy: Selected Papers of Richard Montague, pp. 247-270. New Haven: Yale University Press.

Montague, R. (1974c). Universal grammar. In R. H. Thomason (Ed.), Formal Philosophy: Selected Papers of Richard Montague, pp. 222-246. New Haven: Yale University Press.

Oliver, J. W. (1967). Formal fallacies and other invalid arguments. Mind 76, 463-478.

Sainsbury, M. (1993). Logical Forms. An Introduction to Philosophical Logic. Oxford: Blackwell.

Sainsbury, M. (2001). Logical Forms. An Introduction to Philosophical Logic (2nd ed.). Oxford: Blackwell.

Salmon, W. (1973). Logic (2 ed.). Englewood Cliffs, NJ: Prentice-Hall.

Wengert, R. G. (1974). Schematizing de morgan's argument. Notre Dame Journal of Formal Logic 1, 165-166.

Wittgenstein, L. (1995). Tractatus logico-philosophicus. Frankfurt a. M.: Suhrkamp. 